\title{
Interview
}

\section{Verdacht auf sexuellen Missbrauch - Arzt in der Pflicht?}

\author{
Sieht ein behandelnder Arzt bei Patienten Zeichen eines sexuellen \\ Missbrauchs, so steckt er oft in einem Dilemma: Der Verpflichtung, \\ eine Straftat aufzudecken steht die Gefahr gegenüber, möglicher- \\ weise Unschuldige in strafrechtliche Ermittlung hineinzuziehen. \\ Woran kann sich der Arzt orientieren und wo kann er sich Rat holen?
}

? Wo können Ärzte, die ja nicht täglich mit dem Verdacht oder der Vermutung eines stattgefundenen sexuellen Missbrauchs konfrontiert werden, möglichst schnell fachlich kompetente Beratung oder auch Auskunft über das juristisch korrekte Vorgehen erhalten?

Mützel: Ärzte, die in Bayern tätig sind, können sich jederzeit telefonisch an das Institut für Rechtsmedizin der Universität München wenden. Es gibt eine für Erwachsene errichtete Opferambulanz und seit Neuestem auch eine Kinderschutzambulanz. Über diese Einrichtung können sowohl mündlich als auch schriftlich Beratungen durchgeführt werden (Anm.d.Redaktion:gewaltopferambulanz@ med.uni-muenchen.de). Hinweise dazu finden sich auf der Homepage des Instituts unter www.rechtsmedizin.med.uni-muenchen.de.

? Viele Ärzte haben Bedenken, dass allein bei einer Rückfrage wegen der Vermutung eines sexuellen Missbrauchsfalls bei Behörden automatisch die Staatsanwaltschaft involviert wird. Wie ist hier die Gesetzgebung? Wozu ist der Mediziner verpflichtet? Mützel: Es gibt für Bayern seit dem 16.5.2008 nach Art. 14 Gesundheitsdienst- und Verbraucherschutzgesetz (GDVG) die Verpflichtung, bei Vorliegen gewichtiger Anhaltspunkte auf sexuellen Missbrauch, Misshandlung oder Vernachlässigung dies unter personenbezogenen Daten unverzüglich dem Jugendamt zu melden. Ansonsten unterliegt der Arzt nach \$203 Strafgesetzbuch (StGB) der Schweigepflicht, die er aber unter Umständen nach §34 Strafgesetzbuch - wegen Angst vor Folgetaten - Rechtfertigender
Notstand - umgehen und die Strafverfolgungsbehörde einschalten kann. Erhält der Arzt hierzulande im Rahmen seiner Tätigkeit also Kenntnis über einen sexuellen Missbrauch, ist er zumindest verpflichtet, das Jugendamt einzuschalten.

? Zum 1. Januar 2012 soll bundesweit das neue Kinderschutzgesetz in Kraft treten. Dabei können Ärzte künftig von ihrer Schweigepflicht entbunden werden, wenn es "gewichtige" Hinweise auf eine Kindesmisshandlung in der Familie gibt. Die Ärzte dürfen dann Informationen an das zuständige Jugendamt weitergeben. Sehen Sie darin eine Verbesserung der Rechtssicherheit für die Ärzte und vor allem einen besseren Schutz der Kinder?

Mützel: Eine bundesweite einheitliche gesetzliche Regelung wäre wünschenswert. Damit könnte Ärzten durchaus mehr (Rechts) Sicherheit gegeben werden. Allerdings bleibt davon die Unsicherheit der Ärzte unbetroffen, eine Kindesmisshandlung zu erkennen und zu dokumentieren. Ein Arzt in einer Praxis sollte sich deshalb vorab ein Netzwerk aufbauen, bestehend aus ärztlichen Kollegen, die man zu Rate ziehen kann, sozialen Einrichtungen, Jugendamt und Polizei , die im Falle einer möglichen Kindesmisshand-

? Abschließend noch ein konkretes Beispiel. Ein neunjähriges Mädchen muss einem kleinen ambulant-chirurgischen Eingriff in Narkose unterzogen werden. Dem Anästhesiepfleger fällt beim Einführen eines Zäpfchens ein weiter Anus bei dem narkotisierten Mädchen auf. Die Anästhesisten lung den Arzt unterstützen können.

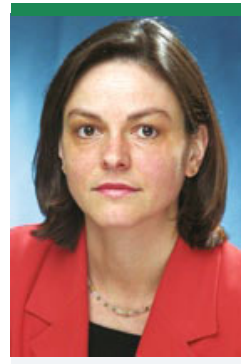

"Eine bundesweite einheitliche gesetzliche Regelung wäre wünschenswert."

PD Dr. med. Elisabeth Mützel Institut für Rechtsmedizin der Ludwig-Maximilians-Universität München

denken an sexuellen Missbrauch, sind sich aber in der Beurteilung des Befundes unsicher. Wie sollen sie vorgehen?

Mützel: In Narkose dilatiert der Analsphinkter häufig so, dass sich dem Anästhesisten, ein weit klaffender Anus darstellt. Anhand dieses Befundes kann keinesfalls auf einen stattgehabten sexuellen Missbrauch geschlossen werden. Ergeben sich in dem Moment keine anderen Hinweise auf das Vorliegen eines möglichen Missbrauchs, sind weitere Schritte nicht einzuleiten.

Das Interview führte Dr. Walther Grohmann, München 